\title{
Epigenetic alterations of the keratin 13 gene in oral squamous cell carcinoma
}

\author{
Kaori Naganuma ${ }^{1,2}$, Mitsutoki Hatta ${ }^{1 *}$, Tetsuro Ikebe ${ }^{2}$ and Jun Yamazaki ${ }^{1}$
}

\begin{abstract}
Background: Epigenetic modifications play important roles in the regulation of gene expression determining cellular phenotype as well as various pathologies such as cancer. Although the loss of keratin 13 (KRT13) is reportedly linked to malignant transformation of oral epithelial cells, the molecular mechanisms through which KRT13 is repressed in oral squamous cell carcinoma (OSCC) remain unclear. The aim of this study is to identify the epigenetic alterations of the KRT13 gene in OSCCs.

Methods: We investigated KRT13 expression levels and chromatin modifications of the KRT13 promoter in the three OSCC cell lines (HSC4, HSC3, and SAS). The expression levels of KRT13 protein and mRNA were analyzed by western blotting and quantitative reverse-transcription polymerase chain reaction, respectively, and the localization of KRT13 protein was detected by immunofluorescence. DNA methylation and histone modifications in the KRT13 promoter were determined by bisulfite sequencing and chromatin immunoprecipitation (ChIP), respectively. For the pharmacological depletion of Polycomb repressive complex 2 (PRC2), cells were treated with 3-deazaneplanocin A (DZNep).
\end{abstract}

Results: KRT13 expression was transcriptionally silenced in the HSC3 and SAS cells and post-transcriptionally repressed in the HSC4 cells, while the KRT13 promoter was hypermethylated in all of the three OSCC cell lines. ChIP analysis revealed that PRC2-mediated trimethylation of Lys 27 on histone H3 (H3K27me3) was increased in the KRT13 promoter in the HSC3 and SAS cells. Finally, we demonstrated that the treatment of SAS cells with DZNep reactivated the transcription of KRT13 gene.

Conclusions: Our data provide mechanistic insights into the epigenetic silencing of KRT13 genes in OSCC cells and might be useful for the development of diagnostic markers and novel therapeutic approaches against OSCCs.

Keywords: Keratin 13 (KRT13), Oral squamous cell carcinoma (OSCC), Polycomb repressive complex 2 (PRC2), Gene silencing

\section{Background}

Epigenetic mechanisms play important roles in the regulation of gene expression and phenotypic plasticity. The addition of a methyl group to the cytosine of a CpG dinucleotide (i.e., DNA methylation) in the promoter region of genes commonly mediates gene repression and acts as a silencing mechanism [1]. Post-translational modifications of histone tails are important regulatory markers for generating transcriptionally active and inactive chromatin. For instance, the trimethylation of Lys 4. on histone $\mathrm{H} 3$ (H3K4me3) is associated with gene activation, while the methylation of H3K27 (H3K27me3)

\footnotetext{
* Correspondence: hatta@college.fdcnet.ac.jp

${ }^{1}$ Department of Physiological Science and Molecular Biology, Fukuoka Dental College, Fukuoka 814-0193, Japan

Full list of author information is available at the end of the article
}

and H3K9 (H3K9me2 and H3K9me3) is often connected to gene repression $[2,3]$. These epigenetic modifications dynamically regulate the chromatin architecture of promoter regions leading to the establishment of gene expression patterns. Polycomb repressive complex 2 (PRC2) comprises four core components (Ezh2, Suz12, Eed, and RbAp46/48) and several other proteins [4]. Ezh2 contains histone methyltransfease activity and plays an important role in the methylation of H3K27 mediated by PRC2. Dysregulation of PRC2 has been linked to several human cancers including lymphoma, squamous cell carcinoma, and breast and prostate cancer [5-9].

Oral squamous cell carcinoma (OSCC) is the most common neoplasm of the oral cavity and has poor clinical outcomes associated with recurrence and metastasis 
[10]. The Keratin 13 (KRT13) gene encodes a type I acidic keratin which is expressed in the differentiated cells of non-cornified stratified squamous epithelia [11-13]. Notably, the disappearance of KRT13 is often seen in OSCC lesions, while KRT13 is expressed in normal noncornified oral mucosa [14-19]. In addition, KRT13-negative OSCC is associated with a high potential for local recurrence [20]. Although the loss of KRT13 is correlated with the cellular transformation of oral epithelial cells, the epigenetic mechanisms by which KRT13 is repressed in OSCCs remain unclear.

In this study, we examined the epigenetic alterations in OSCC cells by focusing on the silencing mechanisms of the KRT13 gene and showed elevated KRT13 promoter DNA methylation and repressive histone modifications in OSCC cell lines. Furthermore, we found a PRC2 inhibitor effective for restoring KRT13 transcription. Our findings provide molecular insights into the epigenetic silencing of the KRT13 gene in OSCC cells as well as important implications for the development of diagnostic markers and novel therapeutic approaches.

\section{Methods}

\section{Ethics statement}

All experiments in this manuscript have been approved by the Fukuoka Dental College Institutional Biosafety Committee.

\section{Cells and drug treatment}

HSC3 and HSC4 cells were cultured as described previously [21]. Immortalized human keratinocyte $\mathrm{HaCaT}$ cells and OSCC-derived SAS cells were maintained in Dulbecco's Modified Eagle's Medium supplemented with $10 \%$ fetal bovine serum, 50 units $/ \mathrm{ml}$ penicillin, and $50 \mu \mathrm{g} / \mathrm{ml}$ streptomycin and maintained at $37^{\circ} \mathrm{C}$ with $5 \%$ $\mathrm{CO}_{2}$. The potent PRC2 inhibitor 3-deazaneplanocin A (DZNep) was purchased from Sigma-Aldrich (St. Louis, $\mathrm{MO})$. Cells were seeded the day before the drug treatment, and DZNep $(10 \mu \mathrm{M})$ was added to the culture medium for $24 \mathrm{~h}$ or $72 \mathrm{~h}$.

\section{DNA methylation analysis}

Genomic DNA was extracted from cells using the NucleoSpin Tissue kit (Macherey-Nagel, Düren, Germany) and subjected to bisulfite modification using the MethylEasy Xceed kit (Human Genetic Signatures, Randwick, Australia) according to the manufacturer's instructions. The primers used for amplification of the KRT13 promoter after bisulfite modification were 5'-TGGAGTAGATGAA GTGCTAAGAAGG-3' and 5'-AACAAAAAGAATGATT CAGAGGGG-3'. Polymerase chain reaction (PCR) was performed with EX-taq DNA polymerase (Takara Bio Inc., Shiga, Japan), followed by TA cloning into a pMD20-T vector (Takara Bio Inc.) and sequencing of individual clones.

\section{Quantitative RT-PCR}

Total RNA was extracted from cells using the NucleoSpin RNA kit (Macherey-Nagel) and reverse transcription was performed using a PrimeScript RT reagent kit (Takara Bio Inc.) according to the manufacturer's instructions. Quantitative reverse-transcription PCR (RT-PCR) was performed on an ABI 7500 real-time PCR system (Applied Biosystems, Carlsbad, CA) using SYBR Premix Ex Taq II (Takara Bio Inc.). The primers used for KRT13 were 5'-GACCGCCACCATTGAAAACAA-3' and 5'-TCCAG GTCATTAGACAGAG-3'. GAPDH was used as a reference gene for normalization. The primers used for GAPDH were 5' -GGAGCGAGATCCCTCCAAAAT-3' and 5'-GG CTGTTGTCATACTTCTCATGG-3'. PCR conditions were as follows: $95^{\circ} \mathrm{C}$ for $30 \mathrm{sec}$, followed by 45 cycles of $95^{\circ} \mathrm{C}$ for $10 \mathrm{sec}$ and $60^{\circ} \mathrm{C}$ for $1 \mathrm{~min}$. The relative standard curve method was used to quantify relative mRNA levels of KRT13 and GAPDH.

\section{Western blotting}

Cells were scraped and resuspended in RIPA buffer (25 mM Tris- $\mathrm{HCl}$ pH 7.4, $150 \mathrm{mM} \mathrm{NaCl}, 0.1 \%$ SDS, 1\% Noidet P-40, 5 mM EDTA, 1\% sodium deoxycholate). Total protein extracts $(5-10 \mu \mathrm{g})$ were resolved by electrophoresis on 4-20\% polyacrylamide-SDS gels and transferred onto polyvinylidene fluoride membranes. The membranes were blocked with 4\% (w/v) ECL Prime blocking agent (GE Healthcare Life Sciences, Backinghamshire, UK) in $0.1 \%$ Tween-TBS and probed with primary antibodies, followed by horseradish peroxidase-conjugated secondary antibodies. The specific antigen-antibody interactions were detected on a LAS-2000 imaging system (Fuji Film, Tokyo, Japan) using an ECL Prime Western Blotting Detection Reagent (GE Healthcare Life Sciences). Quantification of band intensity was performed using ImageJ $1.47 \mathrm{v}$ (National Institute of Health, Bethesda, MD). Relative expression levels were normalized to $\beta$-actin or histone H3. The following primary antibodies were used in this study: anti-keratin 13 (EPR3671; Abcam, Cambridge, UK; dilution, 1:1000), anti-Ezh2 (\#5246; Cell Signaling Technology, Danvers, MA; dilution, 1:1000), anti-Suz12 (\#3737; Cell Signaling Technology; dilution, 1:1000), antihistone H3 (\#4499; Cell Signaling Technology; dilution, 1:1000), anti-trimethyl histone H3 (Lys27) (MAB323B; MAB Institute Inc., Sapporo, Japan; dilution, 1:1000), and anti- $\beta$-actin (sc-47778; Santa Cruz Biotechnology, Dallas, TX; dilution, 1:2000).

\section{Immunofluorescence}

Cells were plated on Nunc Lab-Tek chamber slides (177429; Thermo Scientific, Waltham, MA), incubated for $24 \mathrm{~h}$, fixed with $4 \%$ paraformaldehyde in PBS for $20 \mathrm{~min}$ at $21-25^{\circ} \mathrm{C}$, permeabilized with $0.1 \%$ Triton X-100 for $30 \mathrm{~min}$, and washed three times with PBS. Cells 
were blocked with $1 \%$ bovine serum albumin and $0.1 \%$ Tween 20 in PBS and probed with anti-keratin 13 (EPR3671; Abcam, dilution; 1:100), followed by antirabbit immunoglobulin-G (IgG) antibody conjugated with Alexa Fluor 488 (A-11034; Life Technologies, Carlsbad, CA; dilution, 1:800). Nuclei were counterstained with DAPI (P36935; Life Technologies). Fluorescence imaging was performed and images were captured using a Biorevo microscope (BZ-9000; Keyence, Osaka, Japan).

\section{Chromatin immunoprecipitation}

Cells were fixed in culture medium containing $1 \%$ formaldehyde for $10 \mathrm{~min}$ at $21-25^{\circ} \mathrm{C}$ and incubated in NP-40 buffer (10 mM Tris- $\mathrm{HCl} \mathrm{pH} \mathrm{8.0,} 10 \mathrm{mM} \mathrm{NaCl}$, $0.5 \% \mathrm{NP}-40$ ) for $5 \mathrm{~min}$ at $21-25^{\circ} \mathrm{C}$. Cell were harvested and resuspended in SDS lysis buffer $(50 \mathrm{mM}$ Tris- $\mathrm{HCl}$ pH 8.0, 1\% SDS, 10 mM EDTA), followed by 5-fold dilution in chromatin immunoprecipitation (ChIP) dilution buffer (50 mM Tris- $\mathrm{HCl} \mathrm{pH}$ 8.0, $167 \mathrm{mM} \mathrm{NaCl}, 1.1 \%$ Triton X-100, 0.11\% sodium deoxycholate). Chromatin was sonicated using a Bioruptor (Cosmo Bio Co., Tokyo, Japan) at medium power ten times for $20 \mathrm{sec}$. Ten micrograms of soluble sheared chromatin was incubated overnight at $4^{\circ} \mathrm{C}$ with protein $\mathrm{G}$ magnetic beads (\#9006; Cell Signaling Technology) bound to $2 \mu \mathrm{g}$ anti-histone H3 (\#4620; Cell Signaling Technology), anti-trimethyl histone H3 (Lys4) (\#9751; Cell Signaling Technology), anti-trimethyl histone H3 (Lys27) (\#9733; Cell Signaling Technology), or control IgG (Cell Signaling Technology \#2729), followed by sequential washing with low salt RIPA buffer (50 mM Tris- $\mathrm{HCl}$ pH 8.0, $150 \mathrm{mM} \mathrm{NaCl}$, 1 mM EDTA, $0.1 \%$ SDS, $1 \%$ Triton $\mathrm{X}-100,0.1 \%$ sodium deoxycholate), high salt RIPA buffer (50 mM Tris- $\mathrm{HCl}$ $\mathrm{pH}$ 8.0, $500 \mathrm{mM} \mathrm{NaCl}, 1 \mathrm{mM}$ EDTA, 0.1\% SDS, 1\% Triton X-100, 0.1\% sodium deoxycholate), wash buffer (50 mM Hepes- $\mathrm{KOH} \mathrm{pH}$ 7.5, $500 \mathrm{mM} \mathrm{LiCl,} 1 \mathrm{mM}$ EDTA, 1\% NP-40, 0.7\% sodium deoxycholate), and TE buffer. Immune complexes were then eluted by incubation for $20 \mathrm{~min}$ at $65^{\circ} \mathrm{C}$ with ChIP direct elution buffer (50 mM Tris- $\mathrm{HCl}, \mathrm{pH} 8.0,10 \mathrm{mM}$ EDTA, 1\% SDS), and the cross-linking was reversed by incubating overnight at $65^{\circ} \mathrm{C}$. DNA was purified using the GenElute PCR Clean-up kit (Sigma-Aldrich) and subjected to quantitative PCR on an ABI 7500 real-time PCR system (Applied Biosystems). The primers used for the KRT13 promoter were 5' -TTGTGGGAAACAGAAGTGTAGTTGGC-3' and 5'-GGTGAGAGCAGGATTGAGAGCAGGT -3'.

\section{Statistics}

All values are presented as the means \pm SEM for each group. Statistical analysis was performed using Student's $t$-test to compare the means of two groups or by a oneway analysis of variance followed by Dunnett's post hoc test for more than three groups. $\mathrm{p}<0.05$ was considered significant.

\section{Results}

KRT13 repression by multiple mechanisms in OSCC cells First, we examined KRT13 expression levels in the differentiated OSCC cell line (HSC4), in the poorly differentiated OSCC cell lines (HSC3 and SAS), and in the immortalized human keratinocyte $\mathrm{HaCaT}$ cell line [22-24]. Western blotting showed that KRT13 protein levels were significantly decreased in the HSC4 and HSC3 cells
A

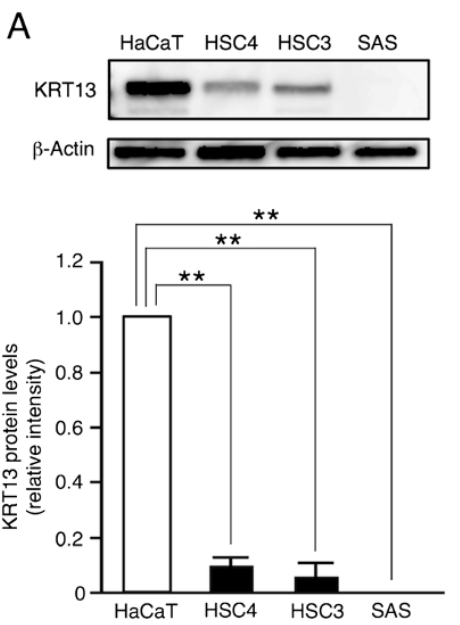

B
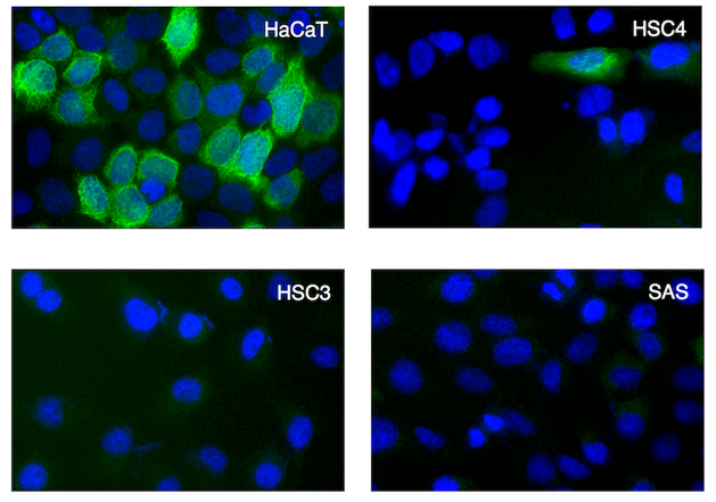

Figure 1 Loss of KRT13 protein in oral squamous cell carcinoma (OSCC) cells. (A) KRT13 protein levels in three OSCC cell lines and HaCaT cells were examined by western blotting. Representative images are shown. Fold change in KRT13 protein was calculated relative to band intensity of the HaCaT cells and normalized to that of $\beta$-actin. The means \pm SEM for each group $(n=4-5)$ are shown. Statistical analysis was performed by a one-way analysis of variance followed by Dunnett's post hoc test. ${ }^{* *} p<0.01$. (B) Expression of KRT13 (green) was analyzed by immunofluorescence staining, and nuclei were visualized with DAPI staining (blue). 
compared with the HaCaT cells, and almost absent in the SAS cells (Figure 1A). Immunofluorescence microscopy revealed high cytoplasmic expression of KRT13 protein in the $\mathrm{HaCaT}$ cells, but not in the OSCC cell lines (Figure 1B and Additional file 1: Figure S1). To investigate whether the reduction in KRT13 protein levels was due to the reduced expression of KRT13 mRNA, we performed quantitative RT-PCR analysis. As shown in Figure 2, KRT13 mRNA levels were significantly decreased in the HSC3 and SAS cells, but not in the HSC4 cells, compared with $\mathrm{HaCaT}$ cells. These results suggest that KRT13 expression is likely to be repressed by translational inhibition or protein degradation in the HSC4 cells and transcriptionally silenced in the HSC3 and SAS cells.

\section{KRT13 promoter methylated in OSCC cells}

Since it has been reported that the methylation status of the promoter region is generally linked to gene repression [1], we examined the DNA methylation status of the KRT13 promoter in the OSCC cells. The CpG sites within the proximal promoter region $(-470$ to -190$)$ were

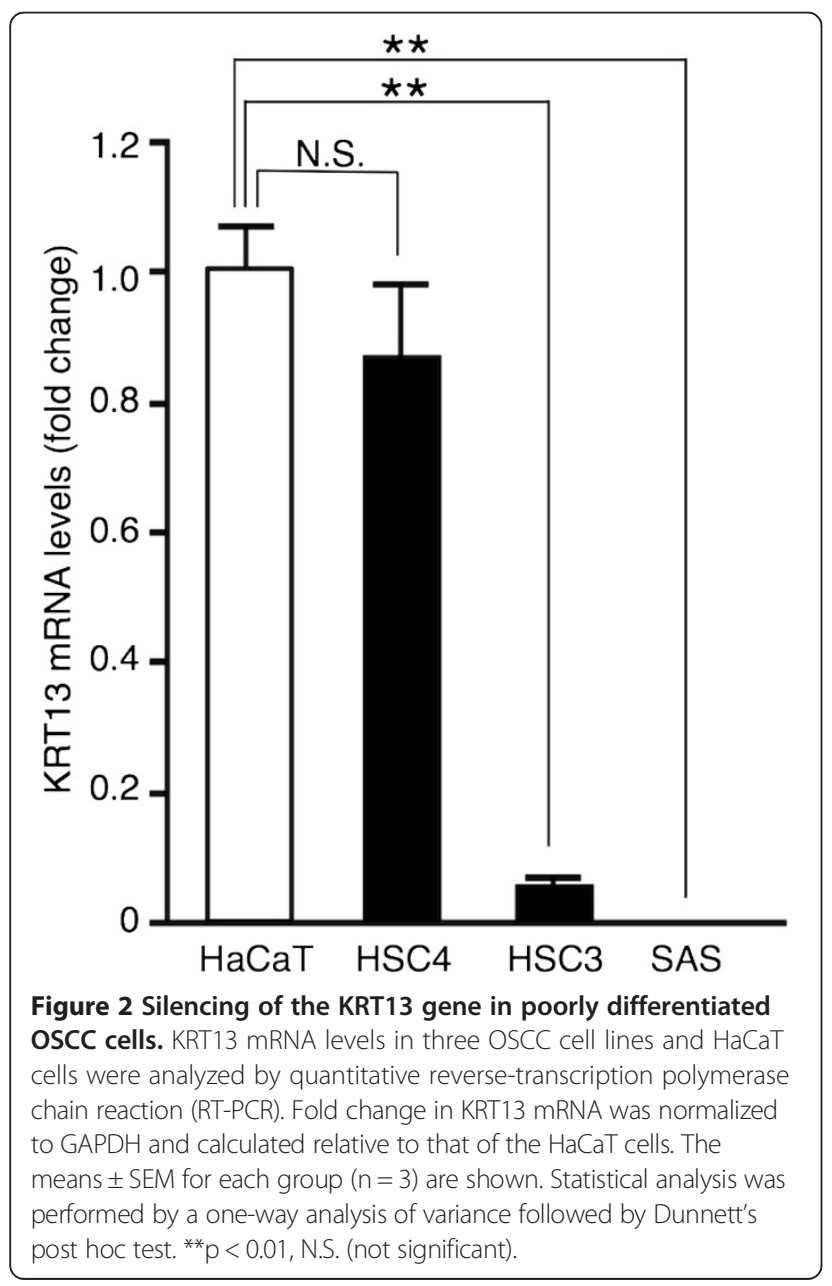

analyzed by bisulfite sequencing (Figure 3A). As shown in Figure 3B, the KRT13 promoter was partially methylated $(<50 \%)$ in the $\mathrm{HaCaT}$ cells and highly methylated $(\geq 90 \%)$ in the three OSCC cell lines (HSC4, HSC3, and SAS). These data show that the KRT13 promoter was aberrantly hypermethylated in all of the examined OSCC cell lines.

\section{Alterations of histone $\mathrm{H} 3$ methylation patterns in the KRT13 promoter in OSCC cells}

DNA methylation of the KRT13 promoter did not correlate with KRT13 gene repression. Thus, it is likely that alterations of histone modifications are responsible for KRT13 gene repression in OSCC cells. It has been reported that $\mathrm{H} 3 \mathrm{~K} 4 \mathrm{me} 3$ is associated with active transcription, while $\mathrm{H} 3 \mathrm{~K} 27 \mathrm{me} 3$ is generally linked to gene repression [2,3]. We therefore examined histone $\mathrm{H} 3$ methylation patterns at the KRT13 promoter in the $\mathrm{HaCaT}$ cells and the three OSCC cell lines. ChIP analysis demonstrated that high levels of H3K4me3 but low levels

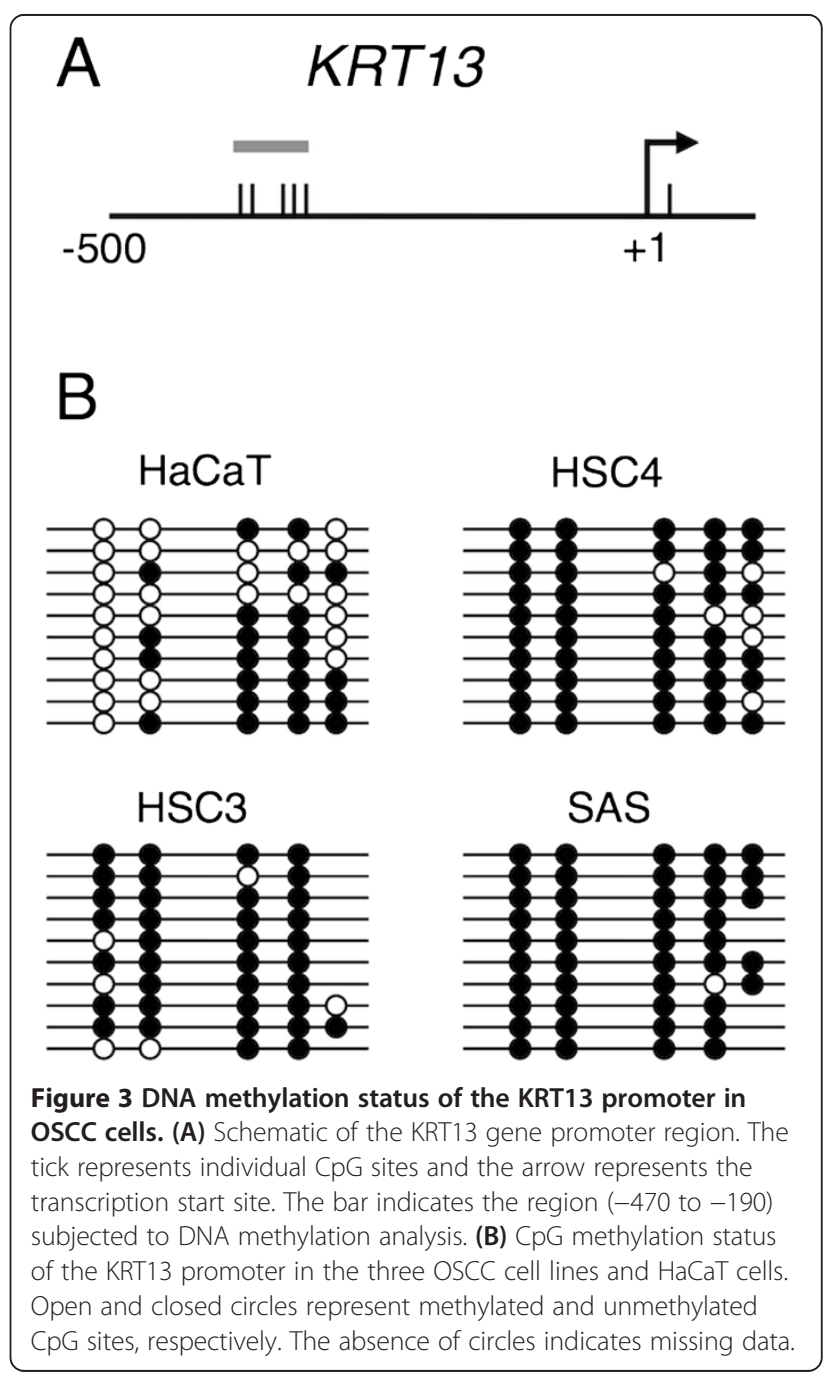


of $\mathrm{K} 27 \mathrm{me} 3$ were detected in the $\mathrm{HaCaT}$ and HSC4 cells, in which KRT13 mRNA was highly expressed. On the other hand, HSC3 and SAS cells showed a histone code switch to high H3K27me3 but low K4me3 (Figure 4A). Additionally, we observed high levels of H3K9me2 and K9me3, and low occupancy of RNA polymerase II (RNAP II) at the KRT13 promoter in the SAS cells compared with the $\mathrm{HaCaT}$ cells (Additional file 2: Figure S2A and B). Since PRC2 is known to play an important role in gene repression through the demethylation of H3K4 and the trimethylation of H3K27 and is linked to human cancer [5-9], we examined the expression levels of PRC2 component proteins (Ezh2 and Suz12) by western blotting. As shown in Figure $4 B$, we found no significant differences in the levels of Ezh2 and Suz12 proteins among the four cell lines. These data suggest that the histone H3 methylation patterns in the KRT13 promoter of the poorly differentiated OSCC cell lines (HSC3 and SAS) are distinct from those of the $\mathrm{HaCaT}$ and HSC4 cells and that the aberrant targeting of PRC2 to the KRT13 promoter may cause this sitespecific alteration.

\section{Reactivation of KRT13 transcription by the PRC2 inhibitor DZNep in OSCC cells}

To determine whether PRC2-mediated transcriptional repression is responsible for KRT13 gene silencing, we examined the effects of the potent PCR2 inhibitor DZNep on KRT13 mRNA expression in the SAS cells. As shown by western blotting (Figure $5 \mathrm{~A}$ ), treatment of the SAS cells with $10 \mu \mathrm{M}$ DZNep for $24 \mathrm{~h}$ decreased the Ezh2 and Suz12 protein levels and H3K27 trimethylation levels, and KRT13 mRNA expression was markedly increased by almost 30-fold after DZNep treatment (Figure 5B). In addition, the H3K27me3 levels in the KRT13 promoter were reduced in the SAS cells treated with DZNep (Figure $5 \mathrm{C}$ ). We then investigated the reversibility of DZNep-induced KRT13 transcription in SAS cells, which had been treated with DZNep for $24 \mathrm{~h}$. KRT13 mRNA levels were increased after additional exposure to DZNep for $48 \mathrm{~h}$, whereas the mRNA levels were reversed for $48 \mathrm{~h}$ after withdrawing DZNep (Figure 6A). As shown in Figure 6B, the Ezh2 and Suz12 protein levels were decreased by DZNep treatment, and increased more than normal levels after drug removal. Taken together, our data suggest that DZNep induces the down-regulation of Ezh2 proteins and reactivates the transcription of KRT13 gene in SAS cells and that the effects of DZNep treatment on SAS cells are not heritable changes.

\section{Discussion}

This study provides molecular insights into the epigenetic silencing of the KRT13 gene in OSCC cells.

\section{A}
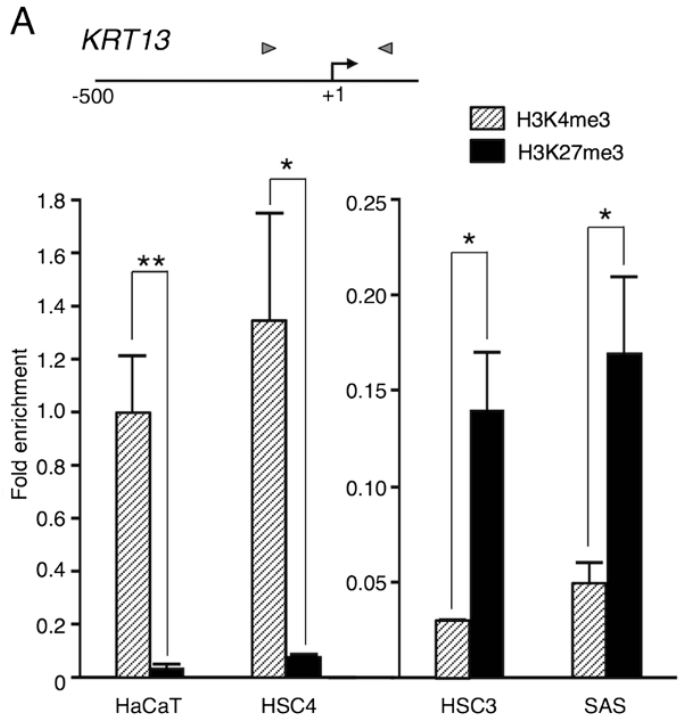

B
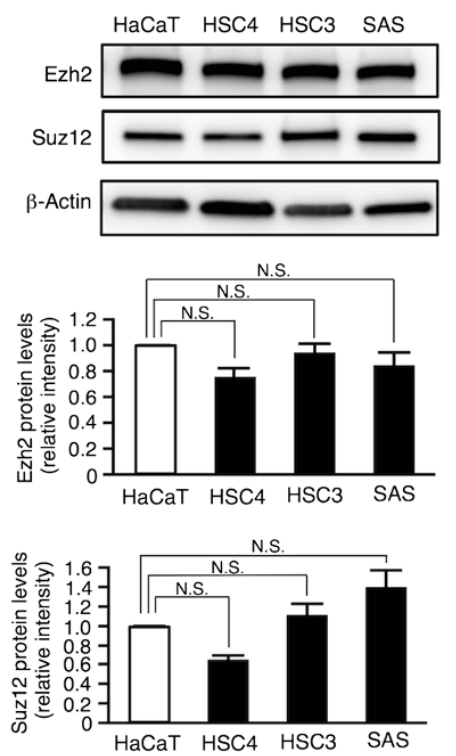

Figure 4 Alterations of histone modifications in the KRT13 promoter in OSCC cells. (A) Schematic of the promoter region of the KRT13 gene. The arrow represents the transcription start site, and arrowheads indicate the primer set used for quantitative PCR. The methylation patterns of histone H3 (H3K4me3 and H3K27me3) in the KRT13 promoter in the three OSCC cell lines and HaCaT cells were examined by chromatin immunoprecipitation (ChIP) assay. Fold enrichment of histone $\mathrm{H} 3$ modifications was normalized to total histone $\mathrm{H} 3$ and calculated relative to that of HaCaT cells. The value of H3K4me3 in the HaCaT cells was set to 1 . The means \pm SEM for each group $(n=3-5)$ are shown. Statistical analysis was performed using Student's $t$-test. ${ }^{*} p<0.05,{ }^{* *} p<0.01$. (B) The levels of PRC2 proteins (Ezh2 and Suz12) were analyzed in the three OSCC cell lines and HaCaT cells by western blotting. Representative images are shown. Fold change in Ezh2 and Suz12 proteins were normalized to $\beta$-actin and calculated relative to band intensity of the HaCaT cells. The means \pm SEM for each group $(n=3)$ are shown. Statistical analysis was performed by a one-way analysis of variance followed by Dunnett's post hoc test. N.S. (not significant). 


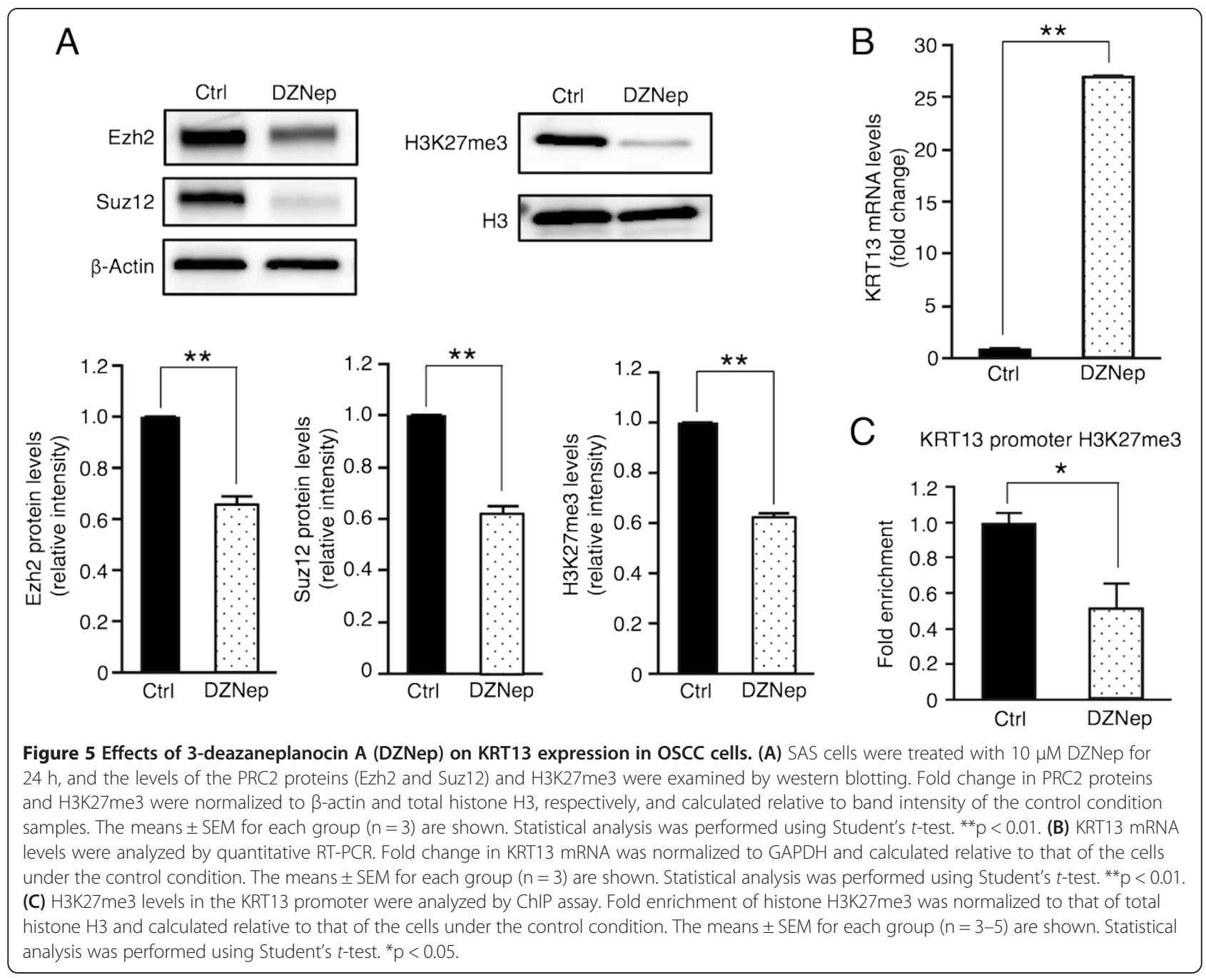

Hypermethylation of $\mathrm{CpG}$ island promoters is known to play a critical role in transcriptional silencing [1], and it has recently been reported that DNA methylation can directly silence genes with non-CpG island promoters [25]. We therefore investigated the DNA methylation status of the KRT13 promoter. According to the defining criteria of CpG islands [26], the KRT13 promoter is classified as a non-CpG promoter. Bisulfite sequencing revealed that the KRT13 promoter was hypermethylated in all of the transformed OSCC cells tested compared with immortalized keratinocytes (Figure 3). Interestingly, Marsit et al. [27] reported that the KRT13 gene was identified as one of the methylated genes associated with aggressive bladder cancer. It is possible that the aberrant DNA methylation patterns of the KRT13 promoter are a hallmark for certain types of cancer.

We evaluated KRT13 expression levels by western blotting, immunocytochemistry, and quantitative RT-PCR. KRT13 protein levels were significantly reduced in all three
OSCC cell lines (Figure 1), while KRT13 transcription levels were decreased in the poorly differentiated OSCC cell lines (HSC3 and SAS), but not in the differentiated OSCC cell line HSC4 (Figure 2). This finding is consistent with a previous immunohistochemistry study using paraffin sections of oral carcinoma in situ [16]. Thus, it is likely that OSCC cells repress KRT13 through multiple mechanisms. Our bisulfite sequencing data suggest that DNA methylation in the KRT13 promoter does not directly mediate transcriptional silencing, and that additional events, such as histone modifications, are required for KRT13 gene silencing in the poorly differentiated OSCC cells.

Epigenetic changes of histone modifications (low H3K4me3 and high H3K27me3) in the KRT13 promoter were identified in the HSC3 and SAS cells (Figure 4). We focused on PRC2-mediated H3K27me3, since it has been reported that $\mathrm{H} 3 \mathrm{~K} 27 \mathrm{me} 3$ and the PRC2 component Ezh2 are involved in squamous cell differentiation [28], and that 

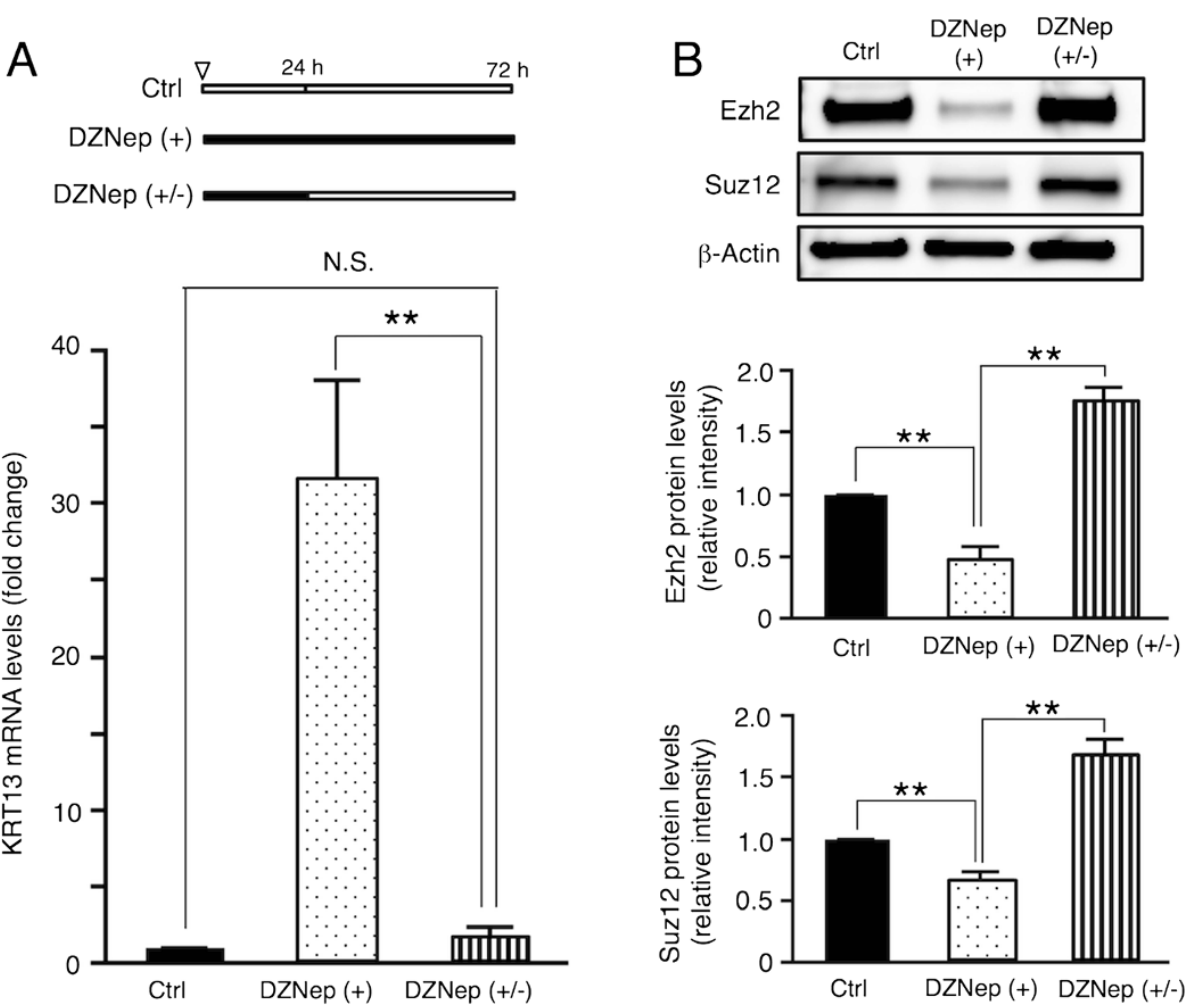

Figure 6 Reversibility of DZNep-induced KRT13 expression in OSCC cells. (A) Schematic of DZNep treatment time. The inverted triangle denotes the starting point. White and black boxes represent incubation without and with $10 \mu \mathrm{M}$ DZNep, respectively. SAS cells were incubated without DZNep for $72 \mathrm{~h}$ (control), with DZNep for $72 \mathrm{~h}$ (DZNep (+)), or with DZNep for $24 \mathrm{~h}$ followed by the removal of DZNep (DZNep (+/-)). KRT13 mRNA levels were analyzed by quantitative RT-PCR. Fold change in KRT13 mRNA was normalized to GAPDH and calculated relative to that of the cells under the control condition. The means \pm SEM for each group $(n=3)$ are shown. Statistical analysis was performed by a one-way analysis of variance followed by Dunnett's post hoc test. ${ }^{* *} p<0.01$, N.S. (not significant). (B) Ezh2 and Suz12 protein levels were examined by western blotting. Representative images are shown. Fold change in Ezh2 and Suz12 proteins was normalized to $\beta$-actin and calculated relative to band intensity of the control condition samples. The means \pm SEM for each group $(n=4)$ are shown. Statistical analysis was performed by a one-way analysis of variance followed by Dunnett's post hoc test. ${ }^{* *} p<0.01$.

deregulation of $\mathrm{PRC} 2$ proteins is closely related to several human tumors [5-9]. To examine the recruitment of PRC2 proteins to the KRT13 promoter in SAS cells, we pharmacologically depleted PRC2 proteins. DZNep was previously reported to deplete the cellular levels of PRC2 proteins and inhibit H3K27 trimethylation [29]. DZNep treatment reduced PRC2 proteins and H3K27me3 levels and reactivated the KRT13 transcription in the SAS cells (Figures 5 and 6). KRT13 is also expressed in the differentiated epithelial cells of the suprabasal layer [11-13], while Ezh2 is expressed in the basal layer of stratified squamous epithelia [28]. In addition to the loss of KRT13, the overexpression of Ezh2 has been reported to correlate with malignant potential and poor prognosis in human OSCCs [6]. Our data suggest that KRT13 is one of the PRC2-repressed target genes in OSCC cells and that the reciprocal expression of KRT13 and PRC2 serves as a useful biomarker for phenotypic characterization of OSCC cells.

\section{Conclusions}

In summary, our study highlights the epigenetic mechanisms of KRT13 silencing in OSCC cells. We identified epigenetic alterations in the KRT13 promoter in OSCC cells and showed that the pharmacological inhibition of PRC2 restored KRT13 expression. To our knowledge, this is the first report on PRC2-mediated epigenetic silencing of KRT13. Our findings may be useful for the development of diagnostic markers and novel therapeutic approaches against OSCCs.

\section{Additional files}

Additional file 1: Figure S1. Cytoplasmic expression of KRT13 protein in the HaCaT cells. HaCaT cells were fixed and probed with anti-keratin 13 (EPR3671; Abcam; dilution, 1:100) or normal rabbit lgG (\#2729; Cell Signaling Technology; dilution, 1:100), followed by anti-rabbit lgG antibody conjugated with Alexa Fluor 488. Expression of KRT13 protein (green) was analyzed using a confocal microscope (LSM710; Carl Zeiss Microlmaging $\mathrm{GmbH}$, Jena, Germany), and nuclei were visualized with DAPI staining (blue). 
Additional file 2: Figure S2. Methylation of $\mathrm{H} 3 \mathrm{~K} 9$ and reduced RNAP ॥ occupancy at the KRT13 promoter in the SAS cells. (A) Methylation of H3K9 were analyzed by ChIP assay using anti-dimethyl histone H3 (Lys9) (\#4658; Cell Signaling Technology), anti-trimethyl histone H3 (Lys9) (MA308B; MAB Institute Inc.). Fold enrichment of histone H3K9me2 and K9me3 were normalized to total histone $\mathrm{H} 3$ and calculated relative to that of the HaCaT cells. The means \pm SEM for each group $(n=3)$ are shown. Statistical analysis was performed using Student's $t$-test. ${ }^{*} p<0.05$, ${ }^{* *} \mathrm{p}<0.01$. (B) RNA polymerase II (RNAP II) occupancy at the KRT13 promoter was analyzed by ChIP assay using anti-RNAP II (clone 4H8; Active Motif, Carlsbad, CA). Fold enrichment of RNAP II was normalized to negative control IgG and calculated relative to that of the $\mathrm{HaCaT}$ cells. The means \pm SEM for each group $(n=3)$ are shown. Statistical analysis was performed using Student's $t$-test. ${ }^{*} p<0.05$.

\section{Competing interests}

The authors declare that they have no competing interests.

\section{Authors' contributions}

$\mathrm{KN}$ and $\mathrm{MH}$ designed and performed the experiments, analyzed the data, and wrote the paper; $\mathrm{Tl}$ designed the experiments and analyzed the data; JY designed the experiments, analyzed the data, and wrote the paper. All authors read and approved the final manuscript.

\section{Acknowledgements}

We thank Drs. Tsuyako Ohkubo, Kazuhiko Okamura, and Kenichi Kato for engaging in helpful discussions. This work was supported by a Grant-in-Aid for Scientific Research (C) from the Japan Society for the Promotion of Science (Grant Number 25462906).

\section{Author details}

'Department of Physiological Science and Molecular Biology, Fukuoka Dental College, Fukuoka 814-0193, Japan. ${ }^{2}$ Department of Oral and Maxillofacial Surgery, Fukuoka Dental College, Fukuoka 814-0193, Japan.

Received: 8 August 2014 Accepted: 16 December 2014 Published: 20 December 2014

\section{References}

1. Deaton $\mathrm{AM}$, Bird $\mathrm{A}: \mathrm{CpG}$ islands and the regulation of transcription. Genes Dev 2011, 25(10):1010-1022.

2. Cheung P, Lau P: Epigenetic regulation by histone methylation and histone variants. Mol Endocrinol 2005, 19(3):563-573.

3. Kouzarides T: Chromatin modifications and their function. Cell 2007, 128(4):693-705.

4. Margueron R, Reinburg D: The Polycomb complex PRC2 and its mark in life. Nature 2011, 469(7330):343-349.

5. Bracken AP, Pasini D, Capra M, Prosperini E, Colli E, Helin K: EZH2 is downstream of the PRB-E2F pathway, essential for proliferation and amplified in cancer. EMBO J 2003, 22(20):5323-5335.

6. Kidani K, Osaki M, Tamura T, Yamaga K, Shomri K, Ryoke K, Ito H: High expression of $\mathrm{EZH} 2$ is associated with tumor proliferation and prognosis in human oral squamous cell carcinomas. Oral Oncol 2009, 45(1):39-46.

7. Gannon OM, Merida de Long L, Endo-Munoz L, Hazar-Rethinam M, Saunders NA: Dysregulation of the repressive H3K27 trimethylation mark in head and neck squamous cell carcinoma contributes to dysregulated squamous differentiation. Clin Cancer Res 2013, 19(2):428-441.

8. Kleer CG, Cao Q, Varambally S, Shen R, Ota I, Tomlins SA, Ghosh D, Sewalt RG, Otte AP, Hayes DF, Sabel MS, Livant D, Weiss SJ, Rubin MA, Chinnaiyan AM: EZH2 is a marker of aggressive breast cancer and promotes neoplastic transformation of breast epithelial cells. Proc Natl Acad Sci U S A 2003, 100(20):11606-11611.

9. Varambally S, Dhanasekaran SM, Zhou M, Barrette TR, Kumar-Sinha C, Sanda MG, Ghosh D, Pienta KJ, Sewalt RG, Otte AP, Rubin MA, Chinnaiyan AM: The polycomb group protein $\mathrm{EZ} 2 \mathrm{H} 2$ is involved in progression of prostate cancer. Nature 2002, 419(6907):624-629.

10. Markopoulos AK: Current aspects on oral squamous cell carcinoma. Open Dent J 2012, 6:126-130.
11. Moll R, Franke WW, Schiller DL, Geiger B, Krepler R: The catalog of human cytokeratins: patterns of expression in normal epithelia, tumors and cultured cells. Cell 1982, 31(1):11-24.

12. Waseem A, Alam Y, Dogan B, White KN, Leigh IM, Waseem NH: Isolation, sequence and expression of the gene encoding human keratin 13 . Gene 1998, 215(2):269-279.

13. Bragulla HH, Homberger DG: Structure and functions of keratin proteins in simple, stratified, keratinized and cornified epithelia. J Anat 2009, 214(4):516-559.

14. Vaidya MM, Borges AM, Pradhan SA, Rajpal RM, Bhisey AN: Altered keratin expression in buccal mucosal squamous cell carcinoma. J Oral Pathol Med 1989, 18(5):282-286.

15. Depondt J, Shabana A, Sawaf H, Gehanno P, Forest N: Cytokeratin alteration as diagnostic and prognostic markers of oral and pharyngeal carcinoas. A prospective study. Eur J Oral Sci 1999, 107:442-454.

16. Ida-Yonemochi H, Maruyama S, Kobayashi T, Yamazaki M, Cheng J, Saku T: Loss of keratin 13 in oral carcinoma in situ: a comparative study of protein and gene expression levels using paraffin sections. Mod Pathol 2012, 25(6):784-794

17. Sakamoto K, Aragaki T, Morita K, Kawachi H, Kayamori K, Nakanishi S, Omura K, Miki Y, Okada N, Katsube K, Takizawa T, Yamaguchi A: Down-regulation of keratin 4 and keratin 13 expression in oral squamous cell carcinoma and epithelial dysplasia: a clue for histopathogenesis. Histopathology 2011, 58(4):531-542.

18. Mikami T, Cheng J, Maruyama S, Kobayashi T, Funayama A, Yamazaki M, Adeola HA, Wu L, Shingaki S, Saito C, Saku T: Emergence of keratin 17 vs. loss of keratin 13: their reciprocal immunohistochemical profiles in oral carcinoma in situ. Oral Oncol 2011, 47(6):497-503.

19. Kitamura R, Toyoshima T, Tanaka H, Kawano S, Kiyosue T, Matsubara R, Goto Y, Hirano M, Oobu K, Nakamura S: Association of cytokeratin 17 expression with differentiation in oral squamous cell carcinoma. J Cancer Res Clin Oncol 2012, 138(8):1299-1310.

20. Yanagawa T, Yoshida H, Yamagata K, Onizawa K, Tabuchi K, Koyama Y, Iwasa S, Shimoyamada H, Harada H, Omura K: Loss of cytokeratin 13 expression in squamous cell carcinoma of tongue is a possible sign for local recurrence. J Exp Clin Cancer Res 2007, 26(2):215-220.

21. Okamoto Y, Ohkubo T, Ikebe T, Yamazaki J: Blockade of TRPM8 activity reduces the invasion potential of oral squamous carcinoma cell lines. Int J Oncol 2012, 40(5):1431-1440.

22. Boukamp P, Petrussevska RT, Breitkreutz D, Hornung J, Markham A, Fusenig NE: Normal keratinization in a spontaneously immortalized aneuploid human keratinocyte cell line. J Cell Biol 1988, 106(3):761-771.

23. Momose F, Araida T, Negishi A, Ichijo H, Shioda S, Sasaki S: Variant sublines with different metastatic potentials selected in nude mice from human oral squamous cell carcinomas. J Oral Pathol Med 1989, 18(7):391-395.

24. Takahashi K, Kanazawa H, Akiyama Y, Tazaki S, Takahara M, Muto T, Tanzawa H, Sato K: Establishment and characterization of a cell line (SAS) from poorly differentiated human squamous cell carcinoma of the tongue. J Jpn Stomatol Soc 1989, 38(1):20-28.

25. Han H, Cortez CC, Yang X, Nichols PW, Jones PA, Liang G: DNA methylation directly silences genes with non-CpG island promoters and establishes a nucleosome occupied promoter. Hum Mol Genet 2011, 20(22):4299-4310.

26. Takai $\mathrm{D}$, Jones $\mathrm{PA}$ : Comprehensive analysis of $\mathrm{CpG}$ islands in human chromosomes 21 and 22. Proc Natl Acad Sci U S A 2002, 99(6):3740-3745.

27. Marsit CJ, Houseman EA, Christensen BC, Gagne L, Wrensch MR, Nelson HH, Wiemels J, Zheng S, Wiencke JK, Andrew AS, Schned AR, Karagas MR, Kelsey KT: Identification of methylated genes associated with aggressive bladder cancer. PLoS One 2010, 5(8):e12334.

28. Ezhkova E, Pasolli HA, Parker JS, Stokes N, Su IH, Hannon G, Tarakhovsky A, Fuchs E: Ezh2 orchestrates gene expression for the stepwise differentiation of tissue-specific stem cells. Cell 2009, 136(6):1122-1135.

29. Tan J, Yang X, Zhuang L, Jiang X, Chen W, Lee PL, Karuturi RK, Tan PB, Liu ET, Yu Q: Pharmacologic disruption of Polycomb-repressive complex 2-mediated gene repression selectively induces apoptosis in cancer cells. Genes Dev 2007, 21(9):1050-1063.

\section{doi:10.1186/1471-2407-14-988}

Cite this article as: Naganuma et al:: Epigenetic alterations of the keratin 13 gene in oral squamous cell carcinoma. BMC Cancer 2014 14:988. 